\section{Cahiers de Narratologie}

Analyse et théorie narratives

$20 \mid 2011$

Voix off et narration cinématographique

\title{
La voix off au féminin : Hiroshima mon amour et Aurélia Steiner
}

Jennifer Cazenave

\section{(2) OpenEdition}

Journals

Édition électronique

URL : http://journals.openedition.org/narratologie/6365

DOI : 10.4000/narratologie.6365

ISSN : 1765-307X

Éditeur

LIRCES

Référence électronique

Jennifer Cazenave, «La voix off au féminin : Hiroshima mon amour et Aurélia Steiner », Cahiers de Narratologie [En ligne], 20 | 2011, mis en ligne le 13 juillet 2011, consulté le 19 avril 2019. URL : http:// journals.openedition.org/narratologie/6365; DOI : 10.4000/narratologie.6365

Ce document a été généré automatiquement le 19 avril 2019

Article L.111-1 du Code de la propriété intellectuelle. 


\title{
La voix off au féminin : Hiroshima mon amour et Aurélia Steiner
}

\author{
Jennifer Cazenave
}

1 En 1958, la maison de production cinématographique Argos Films, qui a produit Nuit et Brouillard (1955), propose à Alain Resnais de réaliser un long métrage sur la bombe atomique. Cette commande s'inscrit dans la continuité de la période dite «courts métrages » de Resnais, à savoir, un ensemble de sept documentaires commandés au jeune réalisateur entre 1948 et 1958 et dont les techniques et les thèmes anticipent les longs métrages à venir: d'un côté, le travelling, la voix off, le mélange de représentations différentes (images d'archives, peinture, sculpture); de l'autre, l'histoire, la mémoire, le lieu. Bien que ces courts métrages s'intéressent à des sujets aussi variés que l'art africain, la Bibliothèque Nationale ou la fabrication du styrène, ils mettent en évidence une nouvelle forme filmique centrée sur la voix off et au travers de laquelle Resnais a réinventé le documentaire commenté ${ }^{1}$.

Dans les documentaires dits «classiques ", le commentaire prend la forme d'une voix off ou voix-sans-corps qui, tout en étant invisible et anonyme, incarne l'omniscience et l'objectivité. «Tel est le pouvoir du off au cinéma, explique François Niney, cet espacetemps indéfini, invisible, extensible qui cerne et hante l'écran, et d'où émane la voix incorporelle du narrateur, du destin, du maitre, des revenants, des savants...qui voit et sait, de son nulle part ailleurs, ce qui se passe ici, devant nos yeux éblouis ${ }^{2} »$. Cette voix que l'on ne voit pas, et qui assimile la représentation et le réel, nous donne à « voir » les images en les recouvrant d'un (seul) point de vue. En outre, cette voix off que l'on retrouve non seulement dans les premiers documentaires "parlés", mais surtout les actualités filmées, s'est vue transformée en véritable outil de propagande pendant la Seconde Guerre mondiale. Si, par la suite, la fonction propagandiste de cette voix perdure dans un contexte politique fortement marqué par la guerre froide et les guerres coloniales, elle devient, dans le domaine du cinéma, le lieu d'une rupture engendrée par un certain nombre de jeunes réalisateurs, qui ont su donner une autre forme au documentaire commenté. 
3 Resnais fait partie de ces jeunes réalisateurs qui, dans l'immédiat après-guerre, remettent en question la vérité par l'image insufflée par la fameuse voix off des documentaires et des actualités filmées - et ceci en repensant la voix dans son rapport à l'image et au spectateur. Or, tout au long de sa période dite « courts métrages ", Resnais réfléchit tout autant sur les limites de la représentation en reliant, justement, les grands traumatismes du vingtième Siècle à des lieux. Dans Guernica (1950), le premier court métrage qu'il consacre au thème de la destruction humaine, l'événement est assimilé au lieu. Si ce titre renvoie à la toile de Picasso et à la ville espagnole détruite par la force aérienne allemande en 1937, « Guernica » est aussi le premier et le dernier mot du commentaire du film : il fait apparaître et disparaitre l'image et symbolise de cette manière le passé et le présent, le traumatisme et sa représentation. Le (nom du) lieu œuvre ici comme un signifiant, une étrange présence-absence - non pas l'événement, mais sa trace - qui témoigne de ce qui ne peut plus être vu.

4 Ainsi, dans Guernica, Resnais se tourne non seulement vers la vision du peintre en nous filmant en noir et blanc sa célèbre toile, mais aussi vers les fragments du monde - un monde marqué par la destruction, l'horreur et la perte humaine. À la composition du film, qui entremêle des toiles, des sculptures, un commentaire et une musique, s'oppose la décomposition du monde, sa destruction, sa fragmentation. Et, de façon similaire à ses courts-métrages précédents, Van Gogh (1948) et Paul Gauguin (1950), où le noir et blanc fait disparaitre les couleurs des toiles afin d'en faire un seul et unique monde, c'est ici à partir de fragments de diverses œuvres de Picasso que Resnais reconstitue un monde brisé. Dans Guernica, la caméra de Resnais filme des œuvres provenant de périodes différentes dans la carrière de Picasso, ainsi que des sculptures contemporaines et des coupures de journaux sur le bombardement. Tandis que le montage permet à Resnais de créer de la continuité à partir d'éléments opposés, ces fragments du monde sont reliés entre eux par une musique composée par Guy Bernard où s'entremêlent divers instruments (tambour, piano, trompette, guitare) et de sons évoquant des avions bombardant une ville. À cette composition musicale s'ajoute une voix off de femme, celle de l'actrice Maria Casarès, qui récite un commentaire poétique écrit par Paul Éluard. Reliant à son tour tous ces fragments d'un monde détruit, cette voix de femme rend présente l'expérience humaine qui sous-tend cette destruction : «Les gens de Guernica sont de petites gens. On a tout lu dans les journaux en buvant son café. Quelque part en Europe une légion d'assassins écrase la fourmilière humaine - quelque part en Europe et c'est à nos frontières. Les balles des mitrailleuses achèvent les mourants, les balles des mitrailleuses jouent avec les enfants mieux que le vent ${ }^{3}$ ».

5 C'est donc à partir d'images disparates et d'une voix off de femme que Resnais a abordé une thématique nouvelle : la destruction humaine. Et si cette mise en scène des horreurs du vingtième Siècle à partir du lieu et de la parole a eu comme point de départ Guernica, elle a par la suite traversé un certain nombre des films modernes de Resnais, notamment son premier long métrage, Hiroshima mon amour. Dans ce film centré sur une voix off de femme, qui se détache sans cesse des images et nous fait voir le visible autrement, la représentation d'un monde anéanti prend la forme d'une rencontre entre des éléments dissemblables: deux villes (Hiroshima et Nevers), deux personnages (une actrice française et un architecte japonais) et deux traumatismes (la bombe atomique et la tonte des femmes à la Libération). Au niveau de la forme, Hiroshima mon amour entremêle des images de matières différentes (fiction et documentaire), brise le rapport entre le son et l'image (asynchronisme) et modifie la représentation du flash-back (glissement du passé 
dans le présent). En outre, bien qu'un choc collectif (la bombe atomique) soit à l'origine de cette fragmentation narrative et formelle, cette dernière se manifeste pleinement au travers d'un choc individuel, à savoir, le traumatisme vécu à Nevers à la fin de la Seconde Guerre mondiale par l'héroïne du film, que l'on connaît que sous la dénomination « Elle », volontairement vague et générique.

Le traumatisme freudien se définit comme un choc émotionnel refoulé, dont le retour opère sur le mode de la répétition, au travers de laquelle le sujet revit l'événement douloureux. Or, ces images - qui doivent, suivant la logique freudienne, être interprétées par les psychanalystes - surgissent tout d'abord de manière incompréhensibles puisque l'événement a été si douloureux que le patient n'a pu se le représenter. Ainsi, le retour témoigne du refoulement en mettant en scène des images que le sujet a vues (et donc enregistrées) sans pourtant le savoir ${ }^{4}$. Dans Hiroshima mon amour, le traumatisme de «Elle» resurgit au travers d'une succession d'analepses qui, en tant représentations fidèles du modèle freudien, reviennent de manière tout d'abord incompréhensibles et ne pourront être pleinement représentées qu'au travers de la parole. Le sujet de Hiroshima mon amour est donc l'histoire - et sa représentation - d'une femme qui, comme le cinéma moderne et comme la ville de Hiroshima, doit se (re)construire à partir de ruines et de fragments, et d'images insoutenables. Le rapport asynchrone entre l'image et la voix redouble donc au travers de la fragmentation du personnage féminin. «Elle » incarne en effet à la fois la décomposition et la recomposition d'un monde brisé car si ses souvenirs (ou flash-backs) rompent la linéarité du récit et bouleversent le présent, sa voix off relie tous les fragments du monde - le document et la fiction, le présent et le passé, Hiroshima et Nevers.

7 De façon analogue au rapport entre la modernité du film et son héroïne, il convient de rappeler le rôle primordial de Duras dans la genèse de Hiroshima mon amour. Resnais, qui avait décidé au moment du choix d'un scénariste de travailler avec une femme, avait d'abord songé à Françoise Sagan et à Simone de Beauvoir, avant de proposer le projet à Duras. Elle a écrit le scénario de Hiroshima mon amour en neuf semaines et, au fil de ce travail d'écriture, elle n'a cessé de correspondre avec Resnais, déjà parti au Japon pour effectuer les repérages. Or, sa collaboration avec Resnais ne se limite pas à ce travail d'écriture. Bien que Duras n'ait pas assisté au tournage à Hiroshima, elle a fait répéter Emmanuelle Riva à Paris, avant que celle-ci ne rejoigne Resnais. De même, elle a enregistré une lecture de son propre texte, que Resnais a écouté lors du tournage au Japon: «Je n'oublierai pas, lui écrit-il, mes étranges journées passées dans ma chambre en compagnie de votre voix et de deux poupées articulées en bois chargées de remplacer Okada et Riva ». Ainsi, comme le résume avec justesse l'universitaire Alain Boillat, «la voix du film, c'est avant tout celle de Duras elle-même ${ }^{5}$ ».

8 Resnais a aussi demandé à Duras de rédiger des notes afin de le guider pour le tournage des flash-backs muets de Nevers dans Hiroshima mon amour. "Faites comme si vous commentiez les images d'un film fait», lui aurait dit Resnais. Intitulées «Les évidences nocturnes ", ces notes, qui constituent l'appendice du scénario du film, anticipent des images encore à venir en décrivant les lieux déserts de Nevers - les remparts, la cave, la forêt. Dans Hiroshima mon amour, ces espaces peuplent les flash-backs muets de Nevers que la voix off de l'héroïne recouvre et creuse en évoquant les images absentes du passé.

Hiroshima mon amour, dont l'entrée dans l'histoire du cinéma moderne est indissociable du flash-back réinventé, rompt avec la compréhension et la chronologie des retours en arrière du cinéma classique en effectuant ce voyage dans le temps à travers la mémoire 
de l'héroïne. Bien que la mémoire soit synonyme de retour en arrière ou représentation (mentale) du passé, la production de souvenirs qu'elle engendre n'est nullement empreinte des limites spatio-temporelles structurant le flash-back classique. La mémoire signifie au contraire la coexistence (ou mélange) de souvenirs de temps et de lieux différents, qui aboutit à une représentation non chronologique du passé. Bien que les flash-backs engendrent ici la réapparition du passé, les images qui surgissent ne peuvent, dans un premier temps, être identifiées; ainsi, telle la confrontation initiale entre l'héroïne et le traumatisme enfoui, le passé apparaît dans un premier temps sans que nous puissions l'identifier en tant que tel. Ainsi, la répétition qui sous-tend le traumatisme chez Freud trouve donc un écho dans les célèbres retours en arrière de Hiroshima mon amour, au travers desquels le passé douloureux et refoulé de l'hérö̈ne (re)surgit.

Le premier flash-back est donc non identifiable en tant que tel et cette image éphémère et muette se greffe sur le récit comme un corps étranger freudien, une image visible mais illisible. Ce n'est que par la suite - à l'occasion du deuxième flash-back - que cette image devient compréhensible non seulement en tant que retour en arrière, mais aussi comme réapparition d'un traumatisme: il s'agit de la mort d'un jeune soldat allemand que l'héroïne a aimé à Nevers pendant la guerre. À l'instar de la fonction centrale occupée par la voix de l'héroïne, ce sont les questions de son amant japonais qui incitent le retour à Nevers. En effet, tandis qu'un léger mouvement de sa main est à l'origine du premier flash-back, une question, qu'il lui pose, nomme a posteriori (une vingtaine de minutes séparent les deux premières analepses) ce corps ensanglanté que nous n'avons pu, jusque là, identifier.

11 «Il était Français, l'homme que tu as aimé pendant la guerre ?», lui demande-t-il, alors qu'une image à nouveau éphémère et silencieuse survient et nous laisse voir un jeune soldat allemand traversant une place. « Non...il n'était pas Français », lui répond la jeune femme, à nouveau visible, ajoutant juste après: «Oui, c'était à Nevers ». Le flash-back reprend et une succession d'images muettes nous montrant des lieux déserts à Nevers - une jeune fille à vélo, une forêt, une cabane, des ruines - nous dévoilent finalement l'histoire d'amour entre la jeune femme et le soldat allemand. Une fois les amants de Hiroshima à nouveau visibles, «Elle » termine son récit : « Et puis, il est mort ». Au lieu de rendre visible - pour la deuxième fois - cette première image du passé, la parole de l'héroïne ramène le spectateur à une temporalité antérieure à la mort de l'Allemand: nous voyons l'héroïne courir à travers les champs de Nevers pour retrouver le jeune soldat ${ }^{6}$.

12 Si la mort de l'Allemand est le premier souvenir à surgir - et le seul du film à être représenté comme image incompréhensible -, c'est qu'elle constitue le point de départ d'une succession d'épisodes douloureux qui se sont déroulés à Nevers et qui ne pourront être révélés qu'au fur et à mesure. "Ça ne veut rien dire, en français, Nevers, autrement? » lui demande sciemment son amant japonais, suggérant de cette manière la présence d'autres images-souvenirs. Et si les questions du Japonais incitent l'héroïne à revenir à Nevers, elles anticipent aussi le véritable lieu du traumatisme : «Tu aurais eu froid, dans cette cave à Nevers si on s'était aimé ?», lui demande-t-il tout de suite après, provoquant alors un long flash-back fragmenté - une quinzaine de minutes entrecoupées par de brefs retours au présent ${ }^{7}$-, qui mène l'héroïne a énoncer deux autres souvenirs enfouis : la tonte qu'elle subit à la Libération, puis son enfermement dans la cave de ses parents, conséquence de son amour interdit ${ }^{8}$. Tout au long $\mathrm{du}$ film, les retours en arrière 
révèlent donc le traumatisme de Nevers au travers d'une succession d'épisodes douloureux, dont l'ordre chronologique serait le suivant : la mort de l'Allemand ; la tonte de l'héroïne ; l'enfermement dans la cave.

Or, dans Hiroshima mon amour, «Elle » ne raconte pas l'histoire de Nevers d'une manière chronologique, mais plutôt sur un mode archéologique où le souvenir le plus enfoui serait le dernier à resurgir. Et si, au moment où l'héroïne se trouve à Hiroshima, la voix off lui permet de revenir sur les lieux de son passé et de les retraverser, ses paroles creusent les lieux afin de faire surgir les souvenirs les plus enfouis car, dans son récit, l'épisode de la tonte est racontée après celui de la cave, même si, chronologiquement, cet événement précède l'enfermement. En outre, tout au long de Hiroshima mon amour, la représentation du passé est tissée à partir d'images de Nevers muettes hantées par les sons de Hiroshima, à la fois la voix off de l'héroïne et les bruits de la ville. Le recouvrement des images du passé par les sons du présent rappelle la répétition freudienne ou l'irruption du refoulé dans les rêves, qui engendre la disparition momentanée de limites spatio-temporelles séparant le passé du présent. Et si ces limites sont, dans le cadre d'une psychanalyse, rétablies a posteriori par la parole, c'est aussi à la voix que revient la charge, tout au long de Hiroshima, de donner un sens aux images du passé.

Il convient de noter que les images de Nevers sont muettes, à l'exception de deux sons : les cloches de Nevers et un cri émis par l'héroïne. Dans La voix au cinéma (1983), Michel Chion consacre un chapitre au cri dans l'histoire du cinéma, une figure de style qu'il nomme - afin de désigner le moment où elle advient dans un film - «le point de cri » et qui, remarque-t-il, est souvent un cri de femme. «L'expression de point de cri vise à souligner que ce n'est pas tellement la substance, la modulation de ce cri qui est importante, que sa place. [...]. Le point de cri est un point d'impensable à l'intérieur du passé, d'indicible à l'intérieur de l'énoncé, d'irreprésentable à l'intérieur de la représentation ${ }^{9}$ ». Impensable, indicible, irreprésentable : ainsi pouvons-nous décrire «le point de cri » dans Hiroshima mon amour. Un épilogue de l'événement le plus indicible ou irreprésentable, ce cri advient à la suite des images de la tonte infamante où nous voyons l'héroïne assise, vêtue d'une robe, le visage sale, alors qu'une foule l'entoure et qu'un homme lui coupe les cheveux; puis, lentement, elle se lève - des cheveux sont encore collés à sa robe -, traverse la foule et quitte la place en montant un escalier.

Après un bref retour à Hiroshima, tout au long duquel la caméra se rapproche de son visage pour aboutir à un gros plan, elle évoque son retour à la maison, le soir même, et cette parole engendre un nouveau retour en arrière. À ce moment-là, nous entendons un cri (de femme) et nous voyons simultanément une femme sortir d'une maison en courant. Cependant, l'origine du cri n'est pas la femme que nous voyons et le cri apparaît comme un champ (sonore) juxtaposé à un contrechamp (visuel). En effet, la femme que nous voyons est filmée depuis le lieu du cri : il s'agit de la mère de l'héroïne, qui court vers sa fille, demeurée jusqu'à présent hors-champ ${ }^{10}$.

16 Apparition purement acoustique, le cri n'est ainsi jamais visible en tant que tel, mais revêt à la place l'apparence d'une autre femme, l'image de la mère, dont les cheveux épais et longs semblent recouvrir momentanément les cheveux tondus de l'héroïne. Ainsi, telle une parole sans lieu, qui surgit une fois l'épisode de la tonte révélée, le cri invisible désigne la représentation de l'absence de la représentation. En effet, si « le point de cri » relève de l'indicible et de l'irreprésentable, c'est qu'il désigne ici non seulement la douleur liée au traumatisme de l'héroïne, mais aussi l'Histoire en elle-même, une histoire qui, en 1959, n'était pas encore racontable : celles des femmes tondues à la Libération. Ce 
châtiment a pris la forme d'un spectacle, qui a même parfois été photographié, car la majorité des femmes ont été tondues en public et donc devant une foule. Cependant, cet événement a longtemps été omis des ouvrages historiques consacrés à la Seconde Guerre mondiale, alors même que la littérature et le cinéma l'évoquaient ${ }^{11}$.

Si Hiroshima mon amour est la première œuvre cinématographique à rendre visible cet événement oublié de l'Histoire, il importe de préciser que le film donne à voir à la fois la tonte et son recouvrement. Ce recouvrement, qui précède la représentation de ce traumatisme, est mis en scène par une succession de flash-backs muets où le passé apparait tout d'abord comme un espace que l'on pourrait nommer - en reprenant une expression de Claude Lanzmann ${ }^{12}$ - un non-lieu de mémoire : un espace quelconque (une forêt, un champ, des ruines), qui témoigne de l'absence des traces de la tonte. Contrairement à la ville de Hiroshima, où les représentations du passé se succèdent au fil d'un court-métrage d'une vingtaine de minutes au début du film, la représentation du traumatisme procède ici à rebours. Les non-lieux de mémoire apparaissent en premier et il revient à la voix off de l'héroïne de creuser ces images champêtres avant d'aboutir au passé traumatique qu'elles recouvrent et duquel il ne subsiste, au moment présent, aucune trace. En outre, bien que Resnais ait eu recours à une reconstruction fictionnelle afin de représenter la tonte et l'enfermement dans la cave, il convient de remarquer que l'héroïne effectue, après qu'elle a relaté ces deux événements - après, donc, que la tonte a été représentée par le biais d'une reconstruction fictionnelle -, un ultime retour en arrière $^{13}$.

Assise sur un banc dans la gare de Hiroshima, elle se souvient donc pour la dernière fois de Nevers; tandis que sa voix off prend ici la forme d'un monologue intérieur, les images de Nevers sont redevenues des non-lieux de mémoire: les images champêtres qui apparaissent sont vides et figées, marquées par l'absence de corps et de mouvement.

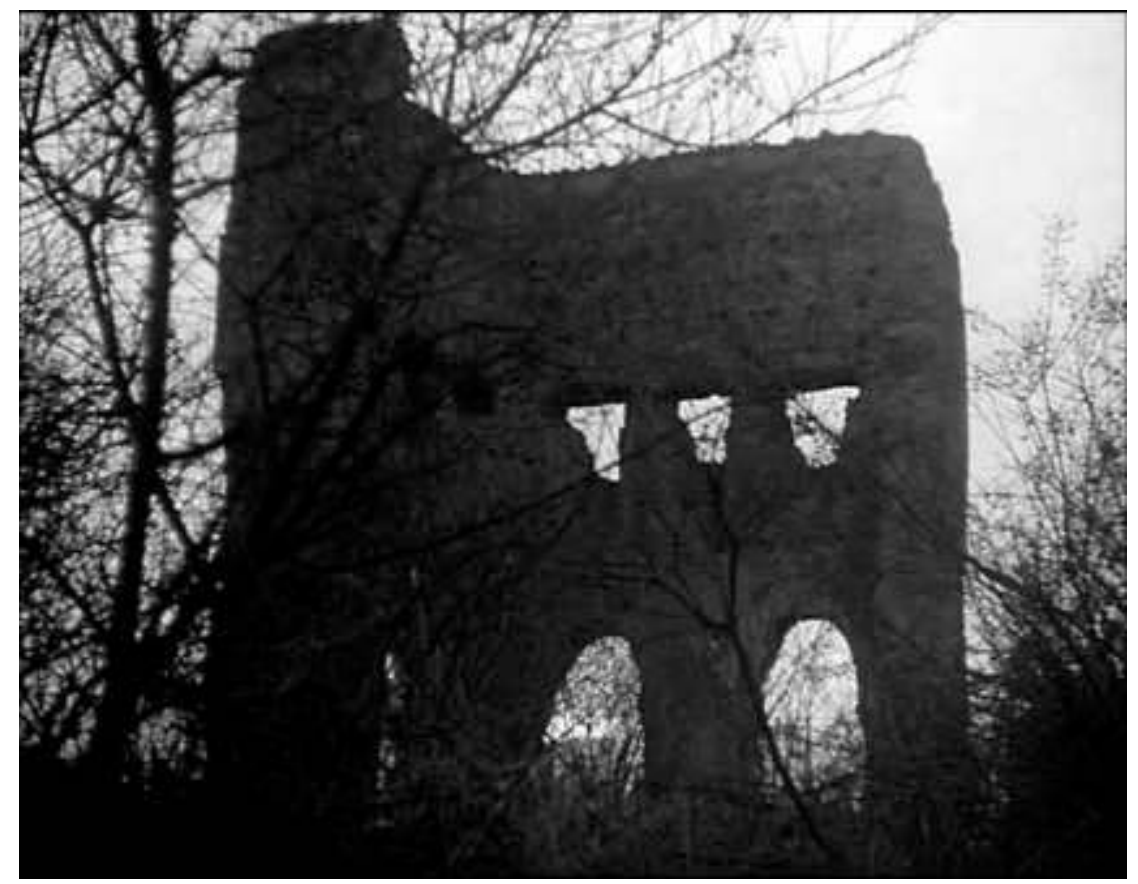




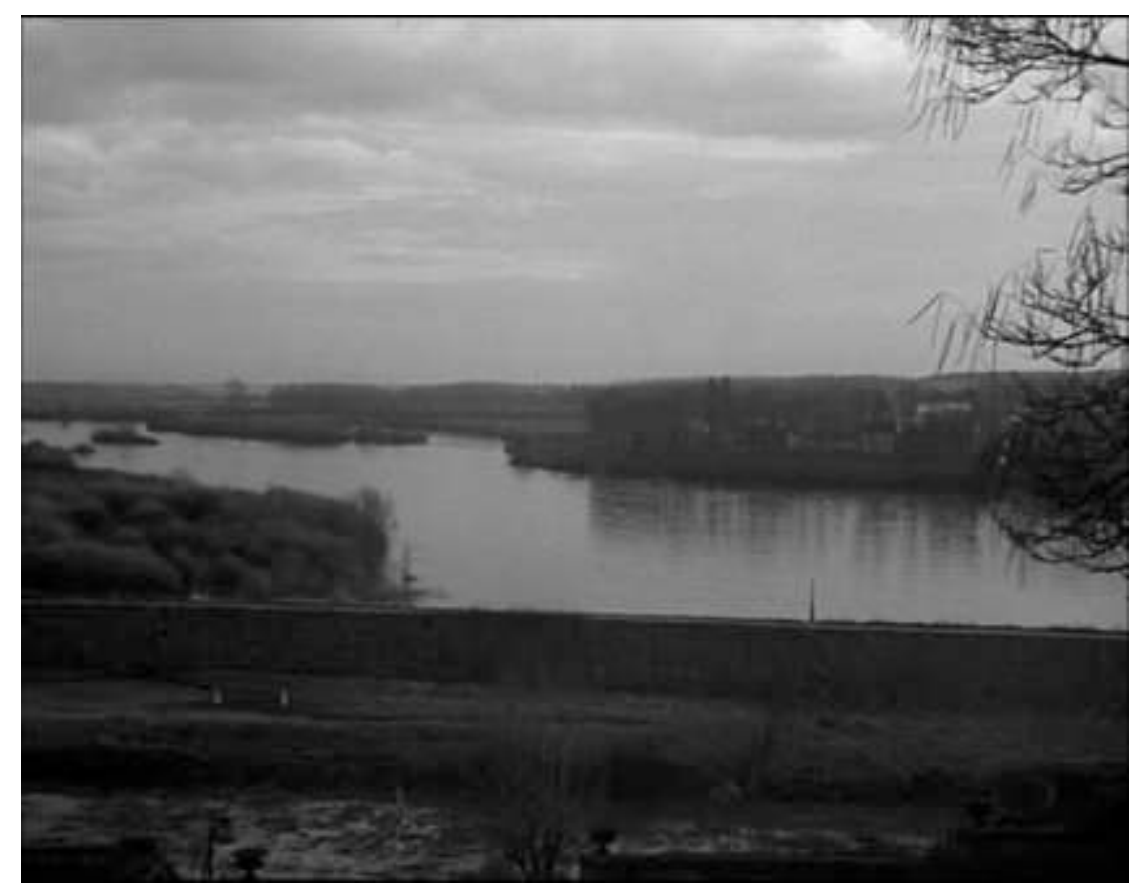

Figures 1 et 2 : Les non-lieux de mémoire de Nevers (Hiroshima mon amour) ["Hiroshima mon amour », production : Argos Films. Tous droits réservés.]

Ainsi, l'histoire de Nevers touche à sa fin car « Nevers » a été racontée et commence petit à petit, depuis cette gare de Hiroshima dans laquelle se trouve l'héroïne, à s'éloigner - à la fois de « Elle ", qui parle d'elle-même à la troisième personne du singulier, et de nous, car ce retour en arrière est le dernier. De même, ces images nous rappellent que la reconstruction fictionnelle de la tonte et de l'enfermement n'aura duré que le temps d'un flash-back: malgré cette mise en scène du passé, ces images se voient à nouveau remplacées par les non-lieux de mémoire de Nevers, qui redoublent ainsi l'absence qui hante toute représentation.

La centralité d'une voix féminine et de lieux de mémoire, qui sous-tendent la représentation du passé dans Hiroshima mon amour, anticipe l'œuvre cinématographique de Marguerite Duras, au fil de laquelle elle a expérimenté, maintes fois, les limites de l'image par le biais de la voix off - en particulier, le parler féminin - et de la désynchronisation de la parole et de l'image. En outre, bien que sa collaboration avec Resnais constitue les prémisses de cette carrière filmique à venir, la remise en cause des limites de la représentation par la voix fait écho à un événement qui a précédé le film de Resnais et le bombardement de Hiroshima, à savoir, le retour des camps de son mari, Robert Antelme, en 1945.

Marguerite Duras a attendu pendant des mois cet homme qui a été déporté en 1944 à la suite de son arrestation par la Gestapo pour ses activités dans la Résistance. "Je me postais dans les gares et devant les entrées des hôtels avec les photos de mon mari. [...] Je ne pleurais pas, j'étais apparemment comme d'habitude sauf que je ne pouvais plus parler du tout ", a-t-elle écrit, des décennies plus tard ${ }^{14}$. Tout en évoquant son mutisme qui a peuplé ces mois d'attente, Marguerite Duras a néanmoins décrit cette attente dans un journal qu'elle a par la suite mis de côté, avant de le publier en 1985 dans un livre intitulé La douleur. Telle l'absence de la parole évoquée ci-dessus, ce journal retrouvé est luimême traversé par l'oubli : «Je n'ai aucun souvenir de l'avoir écrit, explique-t-elle dans un petite texte préambulatoire ajouté pour la publication de La douleur. [...] Comment ai-je 
pu écrire cette chose que je ne sais pas encore nommer et qui m'épouvante quand je la relis $^{15}$ ?». Cette « chose », qui lui semble, au moment de la publication du journal, relever de l'indicible, désigne la rencontre entre le «savoir» et le "voir» des camps : entre l'attente de cet homme envoyé là-bas, puis son retour. Car, au travers de la vue de cet homme, la vision horrifique des camps lui est soudainement apparue.

Dans L'espèce humaine (1947), Robert Antelme relate son expérience de la déportation et livre un témoignage hanté par les limites de la représentation - l'écart irrémédiable entre l'expérience et les mots pour le dire ${ }^{16}$. Juxtaposé à ce récit, le journal de Marguerite Duras prend la forme d'un contre-champ nous permettant de voir, à son retour, cet homme qui a vu. En effet, bien que La douleur relate surtout les mois passés à attendre cet homme - » Je n'ai de place nulle part ici, je ne suis pas ici, mais là-bas avec lui, dans cette zone inaccessible aux autres, inconnaissable aux autres, là où ça brûle et où on tue ${ }^{17}{ }^{\prime}-$, la description du retour d'Antelme est centrée sur l'image de son corps méconnaissable, qui donne lieu à une vision insoutenable. Ainsi, dans $L a$ douleur, Duras n'évoque pas en premier l'image de ce corps, mais le son engendré par son apparition :

J'ai entendu des cris retenus dans l'escalier, un remue-ménage, un piétinement.

Puis des claquements de portes et des cris. C'était ça. C'étaient eux qui revenaient d'Allemagne.

Je n'ai pas pu l'éviter. Je suis descendue pour me sauver dans la rue. [...] Ils étaient arrêtés au palier du premier étage. Il avait les yeux levés.

Je ne sais plus exactement. Il a dû me regarder et me reconnaître et sourire. J'ai

hurlé que non, que je ne voulais pas voir. [...]

Dans mon souvenir, à un moment donné, les bruits s'éteignent et je le vois ${ }^{18}$.

Ce n'est qu'une fois que « les bruits s'éteignent » que Duras arrive à voir cet inconnu à peine en vie qui, pourtant, la regarde, la reconnait et lui sourit. Par ailleurs, alors que Duras décrit, au fil d'une dizaine de pages, le lent retour à la vie du corps d'Antelme, elle commence par évoquer ce qui reste malgré l'horreur - la parole: «Le cœur, lui, continuait à retenir son contenu. Le cœur. Et la tête. Hagarde, mais sublime, seule, elle sortait de ce charnier, elle émergeait, se souvenait, racontait, reconnaissait, réclamait. Parlait. Parlait ${ }^{19}{ }^{\prime}$.

Telle l'insistance de la parole décrite au travers de la répétition du verbe " parler », Duras n'a cessé, par la suite, d'évoquer les camps - et, en particulier, l'extermination des Juifs d'Europe - à la fois dans ses écrits et dans ses films ${ }^{20}$. « Il y a Auschwitz, toute ma vie, oui, affirme-t-elle en 1990. Je le porte encore, rien à faire. C'est là, tout le temps. [...] Les Juifs. Je ne veux pas en parler...C'est l'inconcevable. Des millions assassinés ${ }^{21}$ ». Le souvenir de cet événement «inconcevable » ou irreprésentable, Duras le traite explicitement dans deux courts-métrages sortis en 1979: Aurélia Steiner (Melbourne) et Aurélia Steiner (Vancouver). Ancrés dans le lieu et la parole, ces films représentent l'extermination en couplant des non-lieux de mémoire à deux lettres écrites du point de vue d'une jeune femme de dix-huit ans, Aurélia Steiner, que Duras lit en voix off.

Dans le premier Aurélia Steiner, la caméra se trouve à bord d'un bateau qui remonte la Seine et passe sous de nombreux ponts, filmant, en couleur et à contre-jour ${ }^{22}$, l'eau et ses environs pendant toute la durée du film. Juxtaposée au bruit de l'eau et du moteur du bateau (dont le volume, tout au long du film, ne cesse d'osciller), la voix off de Duras entreprend la lecture d'une lettre destinée à quelqu'un dont l'identité n'est jamais révélée. "Je vous écris tout le temps, toujours ça, vous voyez. Rien d'autre que ça. Rien ${ }^{23}$ ». Il s'ensuit une narration décousue, oscillant entre des questions adressées à ce destinataire inconnu et des descriptions de lieux, eux-mêmes divisés entre des lieux 
quelconques ( Le ciel, au-dessus du fleuve, deviendra noir $\left.{ }^{24} »\right)$ et des lieux qui renvoient directement à l'extermination ( « un camp de Pologne allemande ${ }^{25} »$ ).

Hormis le destinataire, dont la voix off évoque plusieurs fois le regard, la seule présence récurrente dans cette lettre est un chat blanc, qui regarde cette femme au moment où elle écrit. L'apparence de ce chat semble marquée par le souvenir du retour d'Antelme : « Il y a ce chat, maigre, blanc, qui vient me regarder à travers les vitres, les yeux dans les yeux, il me fait peur, il crie, il est perdu, il veut appartenir, et moi je ne veux plus ${ }^{26} »$. Et si ce chat ne cesse de réapparaître, au point de devenir le fil conducteur de cette lettre - » C'est par ce chat maigre et fou, maintenant mort, [...] que je vous atteins ${ }^{27} »-$, cette voix de femme, dont les paroles évoquent d'autres images que celles qui défilent sur l'écran, ne nous révèle son identité qu'à la fin de sa lettre, au moment où la nuit commence à tomber sur la Seine, sur ce lieu que la caméra ne quitte jamais: "Je m'appelle Aurélia Steiner. Je vis à Melbourne où mes parents sont professeurs. J'ai dixhuit ans. J'écris ${ }^{28}$ ".

Dans le second Aurélia Steiner, la caméra, qui fait alterner des travellings et des plans fixes, parcourt des paysages déserts et anonymes, des non-lieux de mémoire filmés en noir et blanc: une plage, des rochers, la mer, une gare, des wagons chargés de troncs d'arbres. Cependant, une première image surgit avant le lieu et la parole : couplée au bruit de la mer, cette image, visible deux fois, précède et succède l'apparition du titre dans le générique $^{29}$. C'est une image que l'on ne saurait identifier, à l'exception de sa ressemblance avec le fond du générique de Hiroshima mon amour ${ }^{30}$. Nous voyons une surface (en pierre) recouverte de fissures et de traces - quelque chose, comme la première image énigmatique de Hiroshima mon amour, qui renvoie à l'irreprésentable et annonce les limites auxquelles va se confronter l'image tout au long d'Aurélia Steiner (Vancouver).

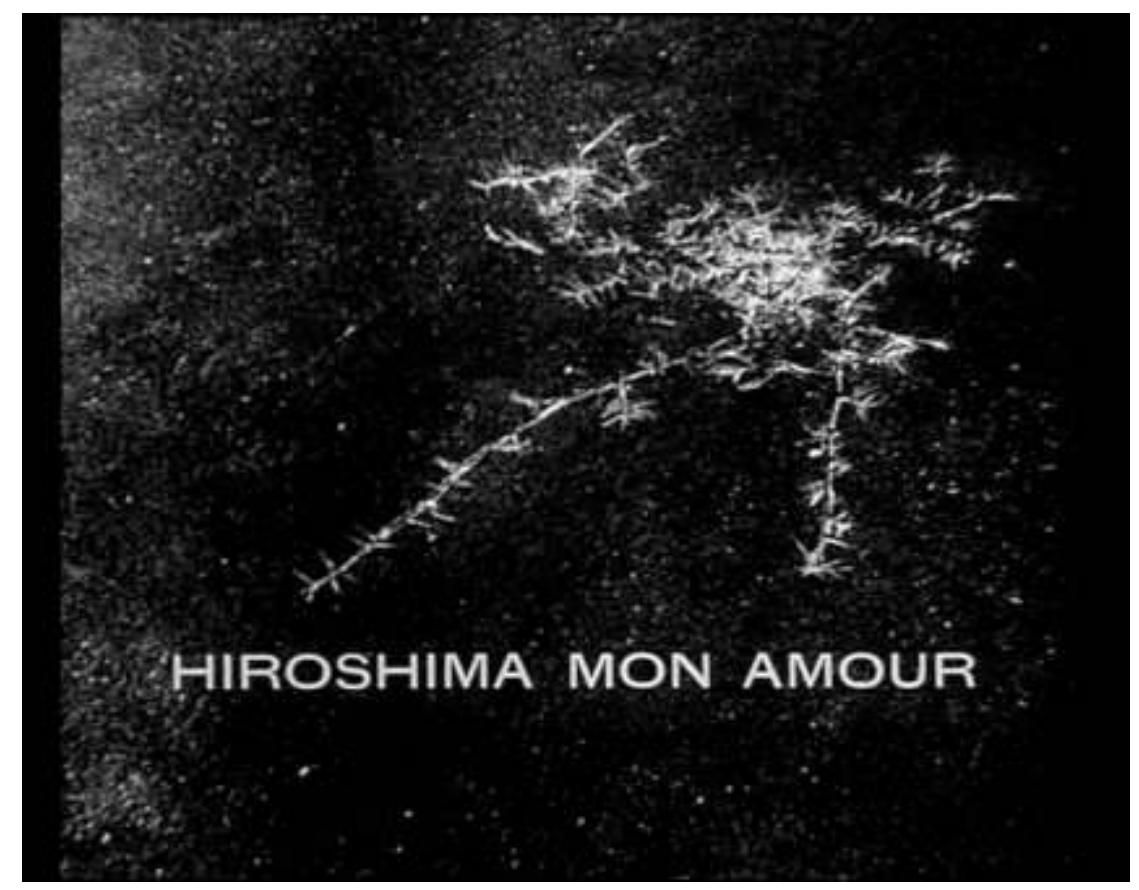

[« Hiroshima mon amour », production : Argos Films. Tous droits réservés.] 


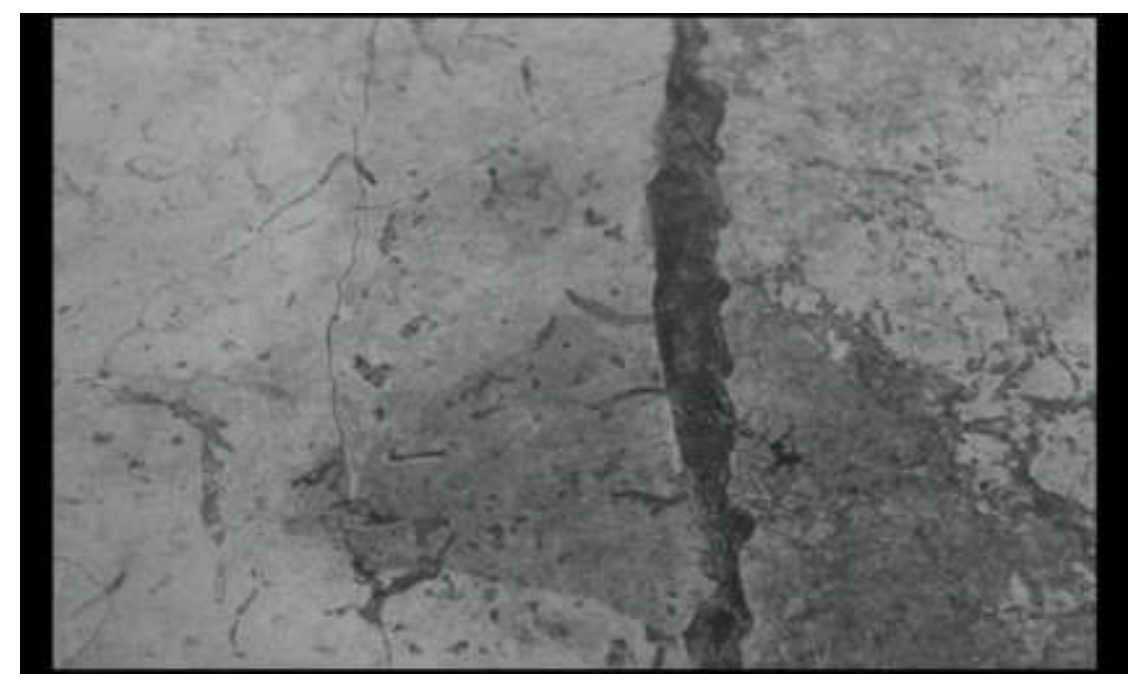

[« Aurélia Steiner (Vancouver) », production : Les films du losange. Tous droits réservés.] Figures 3 et 4 : Les génériques de Hiroshima mon amour et d'Aurélia Steiner (Vancouver)

Si le premier Aurélia Steiner juxtapose un lieu reconnaissable - la Seine ${ }^{31}$ - et un destinataire inconnu, le deuxième juxtapose des lieux quelconques à un homme que la voix off désigne dès les premiers instants comme le père d'Aurélia : « Je m'appelle Aurélia Steiner. Je suis votre enfant. Vous n'êtes pas informés de mon existence. Vous ne pouvez pas me faire signe, la mort vous retient de me voir, je le sais ${ }^{32}$ ». À partir de cette évocation, la voix off tisse un récit entrecoupé par le présent de l'écriture, mais qui nous mène à l'intérieur d'un camp et vers le souvenir de la mort des parents d'Aurélia Steiner. En outre, ce souvenir est indissociable de la présence d'un "rectangle blanc » dans le camp :

Ma mère morte en couches sous les bat-flanc du camp. Brûlée morte avec les contingents des chambres à gaz. Aurélia Steiner ma mère regarde devant elle le grand rectangle blanc de la cour du rassemblement du camp ${ }^{33}$

Dans le rectangle blanc de la cour de rassemblement ma mère Aurélia Steiner distingue encore le pendu voleur de soupe qui gigote au bout de sa corde [...]. Ma mère, dix-huit ans, se meurt. Devant elle, au bout de sa corde, il l'appelle, il crie d'amour fou. Elle n'entend déjà plus ${ }^{34}$.

Tandis que cette deuxième lettre élargit la représentation de l'extermination en situant le souvenir à l'intérieur du camp, l'image du film est néanmoins atteinte - comme semble en témoigner la présence du rectangle blanc - par cette évocation détaillée du désastre. En effet, au passage du noir et blanc s'ajoute - à trois moments différents du film - la disparition de l'image (un écran noir), auquel se succède l'apparition d'un texte écrit à l'encre noire sur une feuille blanche; l'image dans le deuxième Aurélia Steiner est donc momentanément recouverte par l'écriture de Marguerite Duras.

La première séquence à faire apparaitre la parole écrite juxtapose donc trois textes, qui sont séparés par un écran noir qui perdure quelques instants : "Aurélia »; «Aurélia Steiner »; «20009535 ». Ce chiffre, qui renvoie au marquage des corps des déportés dans les camps, entraine le retour à l'image (un champ peuplé d'arbres), qui lui-même donne lieu au premier souvenir de la mère, que nous avons évoqué plutôt. Notons par ailleurs que la voix off de Marguerite Duras perdure, ininterrompue, évoquant à ce moment-là une tempête et une mer agitée. 


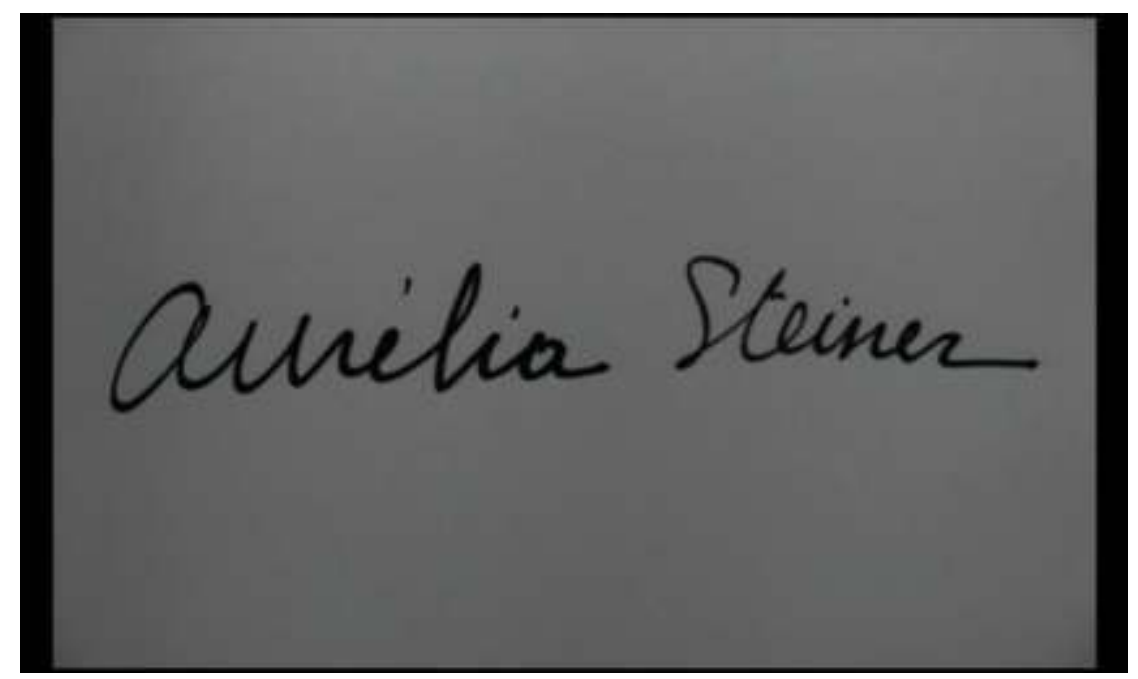

[«Aurélia Steiner (Vancouver) », production : Les films du losange. Tous droits réservés.]

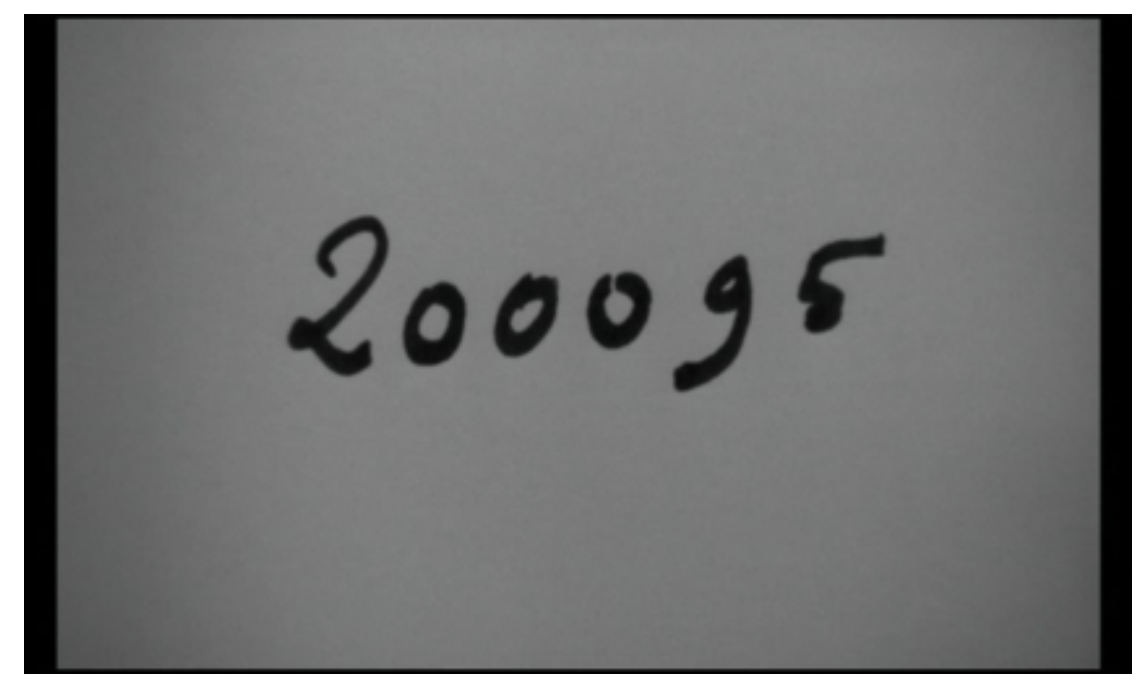

Figures 5 et 6 : Le nom et le chiffre dans Aurélia Steiner (Vancouver)

[«Aurélia Steiner (Vancouver) », production : Les films du losange. Tous droits réservés.]

31 Tandis que la trace écrite (« 200095 ») inscrit la mémoire des camps, alors même que la voix off nous ramène vers un lieu tout autre (la mer), la deuxième apparition de la page écrite dans Aurélia Steiner (Vancouver) est quasiment synchrone avec les paroles prononcées par Marguerite Duras. Bien que nous entendions ici les paroles au moment où nous les voyons, il importe de préciser la présence d'un décalage entre l'image et la parole. Précédé et succédé par deux images noires, le texte apparaît en premier ; ainsi, pendant quelques instants durant lesquels le son de la mer devient de plus en plus faible, avant de céder la place au silence, nous lisons le texte suivant: «Je ne peux rien contre l'éternité que je porte à l'endroit de votre dernier regard celui sur le rectangle blanc de la cour du rassemblement du camp ${ }^{36} »$. Ce n'est qu'une dizaine de secondes plus tard que la voix off de Marguerite Duras se met à réciter le texte, en répétant la première phrase et en l'empreignant du grain de sa voix, qui est ponctuée de peine et de silence : «Je ne peux rien [silence] je ne peux rien [silence] contre l'éternité [silence] que je porte [silence] à l'endroit de votre dernier regard [silence] celui sur le rectangle blanc [silence] de la cour du rassemblement du camp ${ }^{37}$ ». Au moment où la voix évoque «le rectangle blanc", le texte disparaît et l'écran devient noir. La répétition de la première phase, qui fait 
redoubler le sentiment d'impossibilité ( Je ne peux rien »), engendre aussi la division du sujet énonciateur: au «je» du personnage (Aurélia) s'ajoute le «je» de Marguerite Duras, qui improvise cette répétition qui ne s'inscrit nulle part sur l'image ${ }^{38}$, laissant ainsi entrevoir le spectre d'Auschwitz, qui l'a hantée tout au long de sa vie.

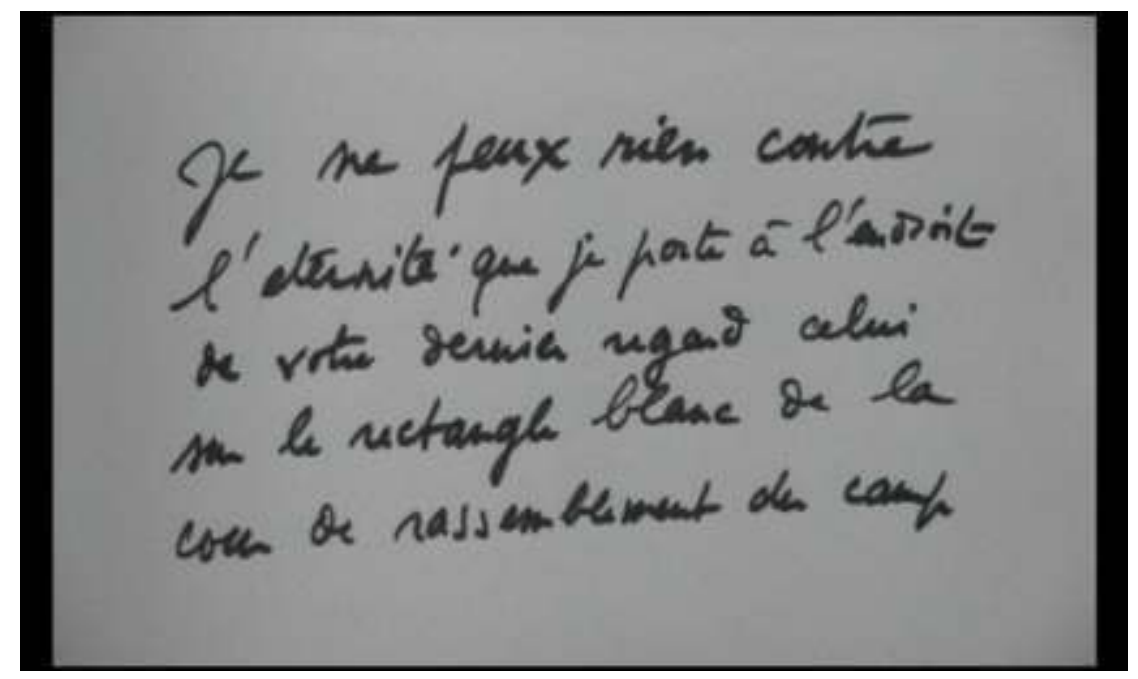

Figure 7 : «Le rectangle blanc » dans Aurélia Steiner (Vancouver)

[«Aurélia Steiner (Vancouver) », production : Les films du losange. Tous droits réservés.]

Dans un texte intitulé "L'image écrite », qu'elle a publié en 1980 dans Les Cahiers du Cinéma, Marguerite Duras révèle l'origine de cette phrase: «[C]'est ma traduction personnelle du livre d'Elie Wiesel, La nuit. Il raconte la mort d'un petit Juif de treize ans qui était si maigre, si léger, qu'il n'arrivait pas à se pendre et qui a gigoté trois jours au bout de sa corde dans la cour du camp. Cette image intolérable depuis toujours, je la vois ainsi : sous le corps de cet enfant, je vois un rectangle blanc ${ }^{39}$ ». Que signifie ce « rectangle blanc ", qui engendre - au moment où Marguerite Duras prononce ces deux mots dans Aurélia Steiner - la disparition du texte et l'apparition de l'écran noir? Bien que «le rectangle blanc » devienne ici le signifiant de l'extermination, ce lieu désigne aussi les limites de la représentation. Car, au cinéma, le rectangle blanc renvoie à la fin de la pellicule, le moment où l'inscription cesse, où l'image n'a plus de lieu. Dans Aurélia Steiner, le rectangle blanc est lié à un « dernier regard » et incarne ainsi le seuil qui sépare l'image et son absence - comme la voix off de Marguerite Duras qui, en prononçant le lieu ou en insérant un rectangle blanc, fait basculer l'image vers le néant ou l'obscurité, vers une image (un écran noir) qui ne donne rien à voir.

Vers la fin d'Aurélia Steiner, la première séquence écrite du film réapparait, à l'exception du chiffre : «Aurélia [écran noir] Aurélia Steiner [écran noir ${ }^{40}$ ] . L'inscription du nom est couplée à un fragment de la lettre où le mot « nom » est répété plusieurs fois :

Parfois il dit le nom tout entier. Parfois il dit seulement le prénom. Parfois le nom seul. Il ne sait plus dire aucun autre mot. Il les dit dans les baisers, les lèvres contre la peau, il les crie, il les appelle à l'intérieur du corps, contre la bouche, contre le mur. Parfois il s'immobilise dans une contention qui la fait gémir, alors il perd la mémoire des noms dirait-on, et puis tout bas, de nouveau il les dit dans un effort douloureux...

Alors que l'image inscrit le nom ("Aurélia Steiner») dans le visible, la voix ne nomme jamais ce nom, le désignant - par le biais de cette absence - comme indicible. Cependant, la voix est hantée par ce «nom» qu'elle ne cesse de répéter. Telle la présence du mot 
allemand Stein - signifiant pierre - dans le nom d'Aurélia Steiner, la voix cherche à inscrire le nom imprononçable - qui fait redoubler les limites de la représentation -, tandis que l'écran devient sa seule inscription possible.

La représentation cinématographique de l'extermination chez Marguerite Duras semble ici anticiper - de façon analogue aux non-lieux de mémoires de Nevers - la démarche de Claude Lanzmann dans Shoah (1985). En effet, dans Aurélia Steiner (Vancouver), l'apparition du nom écrit est précédée quelques instants plutôt de la phrase suivante : «Je lui dit : je vais vous donner un nom ", une phrase qui fait écho à l'épigraphe de Shoah, qui est tiré du livre d'Isaïe dans la Bible : «Et je leur donnerai un nom impérissable (Isaïe, 56, 5) ». Or, si Shoah a par la suite nommé l'événement en France comme à l'étranger, lorsque Marguerite Duras réalise Aurélia Steiner, l'extermination n'était pas encore désignée par un seul et unique nom: on parlait de «l'Holocauste» ou «d'Auschwitz» ou "des camps ». Ce n'est qu'après Shoah que l'événement a été véritablement nommé en France : le nom est devenu la Shoah, un mot hébreu, cette langue étrangère qui fait résonner l'opacité désignée, dans Aurélia Steiner, par « le rectangle blanc». Alors que Shoah tisse une représentation de l'extermination à partir de l'absence -l'omission d'images d'archives, l'omniprésence des non-lieux de mémoire -, les deux films de Marguerite Duras sont, quant à eux, traversés par le non-représenté : des paysages déserts que la caméra ne cesse de parcourir, ainsi que la voix off de Duras, qui désigne des images invisibles.

Tout en anticipant la représentation de l'extermination à venir dans Shoah, les deux Aurélia Steiner font inévitablement écho aux flash-backs muets de Nevers dans Hiroshima mon amour - à ces espaces déserts, quelconques, qui recouvrent un passé dont il ne reste aucune trace et qu'une voix off de femme vient nous révéler. La réflexion placée au centre des films de Resnais et Duras - et par la suite l'œuvre monumentale de Lanzmann reposent donc sur un paradoxe : comment rendre visible ce qui se refuse au regard? En somme, voilà résumée la question fondamentale du cinéma et vers laquelle Hiroshima mon amour et le dyptique Aurélia Steiner ne cessent - en couplant le lieu et la parole - de graviter : que peut-on voir et comment le voir?

\section{NOTES}

1. Les sept courts métrages sont : Van Gogh (1948), Paul Gauguin (1950), Guernica (1950), Les Statues meurent aussi (1953, coréalisé avec Chris Marker), Nuit et brouillard (1955), Toute la mémoire du monde (1956), et Le Chant du Styrène (1958).

2. François Niney, Le documentaire et ses faux-semblants, Paris, Klincksieck, 2009, pp. 115-116.

3. Guernica dans Hiroshima mon amour (DVD 2), Argos Films/Arte Vidéo, 2004, 04"09' - 04"37'.

4. Pour une analyse freudienne de Hiroshima mon amour, voir le livre de Cathy Caruth, Unclaimed Experience. Trauma, Narrative, and History, Baltimore (Maryland), The Johns Hopkins University Press, 1996, pp. 25-56.

5. Alain Boillat, Du Bonimenteur à la voix-over : voix-attraction et voix-narration au cinéma, Lausanne, Éditons Antipodes, 2007, p. 455. 
6. Hiroshima mon amour (DVD 1), Chapitre 3, 37"18' - 40"06'.

7. Contrairement à ce troisième flash-back, les deux premiers retours en arrière se définissent par leur rapidité : le premier dure à peine une minute et le second moins de trois minutes.

8. Hiroshima mon amour (DVD 1), Chapitres 4 et 5, 42" 50 ' - 59" 36 '.

9. Michel Chion, La voix au cinéma, Paris, Cahiers du Cinéma, 1982, p. 68. Si cet ouvrage est aujourd'hui une référence pour les études portant sur la voix au cinéma, il convient de rappeler que ce champ d'étude s'est développé au milieu des années Soixante-dix, comme en témoigne la parution en 1974 du livre de Pascal Bonitzer, Le regard et la voix (1976). En outre, dans son introduction, Chion relie ce tournant dans les études cinématographiques à la fois à un courant féministe structuré autour de la voix et à l'œuvre de cinématographique de Marguerite Duras, qui n'a cessé de repenser les limites de la représentation à partir de la voix (La voix au cinéma, pp. 11-12).

10. Hiroshima mon amour (DVD1), Chapitre 4, 51"31' - 53"10'.

11. Les premières recherches ne sont apparues qu'à la fin des années Quatre-Vingt en France. Voir, en particulier, l'ouvrage de Fabrice Virgili, La France virile: des femmes tondues à la Libération, Paris, Éditions Payot et Rivages, 2004.

12. En effet, ces espaces, qui recouvrent les images absentes du passé et, à ce titre, font redoubler l'absence qui hante toute représentation, anticipent ce que Claude Lanzmann a nommé (en modifiant la formule de Pierre Nora), après la sortie en 1985 de son film monumental Shoah, les non-lieux de mémoire: à savoir, les lieux mêmes de l'extermination, tels que les camps déserts de Treblinka ou Chelmno - des lieux marqués avant tout par la disparition des traces du passés; des lieux déconnectés ou vidés de l'horreur que leurs noms incarnent néanmoins. En outre, tout au long de Shoah, ces lieux marqués avant tout par la disparition des traces sont couplés à la voix off des témoins qui évoquent les images absentes du passé.

13. Hiroshima mon amour (DVD1), Chapitre 7, 1h17" 37 ' - 1h18" 58 '.

14. Marguerite Duras, «Je me souviens », Les yeux verts, Paris : Cahiers du cinéma, 1996, p. 34. Ce livre reprend la totalité des textes de Marguerite Duras qui ont été publiés dans le numéro de juin 1980 ( $\left.n^{\circ} 312 / 313\right)$ des Cahiers du cinéma. C'est elle qui avait entièrement conçu, écrit et mis en page ce numéro des Cahiers.

15. Marguerite Duras, La douleur, Paris, Gallimard, 1985, p. 12. Ces impressions, écrites en 1985, précèdent le journal dans La douleur et figurent aussi sur la quatrième de couverture.

16. Dans l'avant-propos de son livre, Robert Antelme écrit: «Et dès les premiers jours cependant, il nous paraissait impossible de combler la distance que nous découvrions entre le langage dont nous disposions et cette expérience que, pour la plupart, nous étions encore en train de poursuivre dans notre corps. Comment nous résigner à ne pas tenter d'expliquer comment nous en étions venus là ? Nous y étions encore. Et cependant c'était impossible. À peine commencions-nous à raconter, que nous suffoquions. À nous-mêmes, ce que nous avions à dire commençait alors à nous paraître inimaginable» (Robert Antelmne, L'espèce humaine [1947], Paris, Gallimard, 1978, p. 9).

17. Duras 1985, op. cit., p. 62.

18. Ibid., p. 68-9.

19. Ibid., p. 73.

20. Sur le personnage du "Juif durassien», voir l'article d'Hélène Merlin-Kajman, «La communauté judaïsée de Marguerite Duras " dans Marguerite Duras, Paris, Éditions de l'Herne, 2005, pp. 60-68. Sur la centralité d'Auschwitz dans l'œuvre littéraire et cinématographique de Marguerite Duras, voir l'ouvrage de Claire Cerasi, Marguerite Duras de Lahore à Auschwitz, ParisGenève : Champion-Slatkine, 1993.

21. Marguerite Duras, « Des années entières dans les livres » (Entretien avec Marguerite Duras), Les Inrockuptibles, n²1-22, février-mars 1990, p. 115. 
22. «On a tourné Aurélia (Melbourne) à contre-jour, explique-t-elle. À contre-jour, on ne voit pas le visage, humain, on ne voit pas sa forme » (Marguerite Duras, La couleur des mots, Paris, Benoît Jacob, 2001, p. 184).

23. Marguerite Duras, Aurélia Steiner (Melbourne), DVD, Benoît Jacob Vidéo, 2007, 00"58' - 01"08'.

24. Ibid., 08" 47 ' - 08" 52 '.

25. Ibid., 04" 55 ' - 04" 58 '.

26. Ibid., 03" 30 ' - 03" 50 '.

27. Ibid., 24"08'-24"21'.

28. Ibid., 24"47' - 24" 57 '.

29. Marguerite Duras, Aurélia Steiner (Vancouver), DVD, Benoît Jacob Vidéo, 2007, 00"16' - 00"53'.

30. Alain Resnais, Hiroshima mon amour (DVD 1), Chapitre 1,00"2'.

31. «Paris, explique Marguerite Duras, a donné beaucoup de ses Juifs. Les Juifs de Paris valaient trois cent francs par tête. Je pensais, comme ça, à un mouvement général dans lequel se serait perdue Aurélia Steiner » (Duras, La couleur des mots, p. 184).

32. Aurélia Steiner (Vancouver), 07"36' - 08"16'.

33. Ibid., 17"18' - 17" 49 '.

34. Ibid., 23"39' - 24" 31 '.

35. Ibid., 15" 42 ' - 17"14'.

36. Ibid., 19"15' - 19" 26 '.

37. Ibid., 19"27'- 19" 48 '.

38. Cette répétition n'apparaît pas non plus dans le texte du film que Marguerite Duras a publié dans le recueil Le Navire Night, Paris, Gallimard, 1986, p. 133.

39. Duras, «L'image écrite », Les yeux verts, p. 143.

40. Op. cit., $42 " 20$ ' - 43" 25 '.

\section{RÉSUMÉS}

Une étude comparée du grand film moderne d'Alain Resnais, Hiroshima mon amour (1959), et du dyptique de Marguerite Duras, Aurélia Steiner (1979), laisse entrevoir une filiation entre deux films que vingt ans séparent et où s'entrecroisent un certain nombre de fils conducteurs : le lieu et la parole ; l'histoire et la mémoire ; la voix-off au féminin et l'irreprésentable. Il s'agira donc de penser ces deux œuvres cinématographiques à partir du rapport entre le parler féminin et la représentation du passé, soit la tonte des femmes au moment de la libération dans Hiroshima mon amour, soit l'extermination des Juifs d'Europe dans Aurélia Steiner.

\section{INDEX}

Mots-clés : histoire, lieu, mémoire, représentation, Shoah

Index géographique : France 


\section{AUTEUR}

\section{JENNIFER CAZENAVE}

Titulaire d'un double doctorat en cinéma (Université Paris-Diderot et Université de Northwestern), Jennifer Cazenave a récemment soutenu une thèse sur la représentation des femmes dans Shoah (1985), ainsi que dans les rushes du film. 